\title{
Innovative Applications of Advanced Solar Thermal Technologies Using Phase Change Materials
}

\author{
Ben Xu $\left(\mathbb{D},{ }^{1}\right.$ Juan Ordonez, ${ }^{2}$ Zhonghao Rao $\mathbb{D}^{\circ},{ }^{3}$ Xinhai Xu $\mathbb{D}^{4},{ }^{4}$ and Hamidreza Shabgard ${ }^{5}$ \\ ${ }^{1}$ Department of Mechanical Engineering, The University of Texas Rio Grande Valley, Edinburg, TX 78539, USA \\ ${ }^{2}$ Department of Mechanical Engineering, Florida State University, Tallahassee, FL 32306, USA \\ ${ }^{3}$ School of Electrical and Power Engineering, China University of Mining and Technology, Xuzhou, Jiangsu 221116, China \\ ${ }^{4}$ School of Mechanical Engineering and Automation, Harbin Institute of Technology, Shenzhen, Guangdong 518055, China \\ ${ }^{5}$ Department of Aerospace and Mechanical Engineering, The University of Oklahoma, Norman, OK 73019, USA \\ Correspondence should be addressed to Ben Xu; ben.xu@utrgv.edu
}

Received 22 March 2018; Accepted 25 March 2018; Published 27 May 2018

Copyright (C) 2018 Ben Xu et al. This is an open access article distributed under the Creative Commons Attribution License, which permits unrestricted use, distribution, and reproduction in any medium, provided the original work is properly cited.

Recently, phase change material (PCM) have attracted a lot of attentions in energy storage and heating/cooling applications, because of its ability of storing more energy compared with the same amount of sensible storage material, but the intrinsically low thermal conductivity of PCM has restricted its broad applications; as a result, various heat transfer enhancement technologies have been developed [1]. Currently, PCMs have been widely applied in heating/ cooling and heat recovery systems at low and medium temperature range $\left(<200^{\circ} \mathrm{C}\right)$, but the integration of PCM with solar thermal energy is not well documented. Furthermore, most of the PCMs are not thermally or chemically stable at high or extremely high temperature $\left(>200^{\circ} \mathrm{C}\right)$; as a result, it is critical to develop proper engineering ways to incorporate PCM at high temperature for solar thermal applications [2]. Currently, most of the solar thermal applications are in low and medium temperature range; limited information has been reported for high and extremely high-temperature applications due to the limitations of heat transfer fluid, especially for power energy [3].

This special issue is intended to address the integrated solutions using PCMs in various temperature ranges. It is of great interests for researchers and scientists in the fields of mechanical engineering, materials science/engineering, chemical engineering, environmental engineering, and so forth. A brief description and salient results of these papers are given below.
L. Liang and X. Chen prepared and tested a new cold storage phase change material eutectic hydrate salt by adding a nucleating agent and thickener. The physical properties were studied, and the experimental results showed that adding a nucleating agent can effectively improve the nucleation rate and nucleation stability.

$\mathrm{H}$. Li et al. proposed a general design framework for solar water heater (SWH) design using a machine learning-based high-throughput screening (HTS) method, and they used the design of water-in-glass evacuated tube solar water heater (WGET-SWH) as an example; it is expected that this proposed design framework can cost effectively design and optimize a solar thermal system.

S. Z. Farooqui experimentally investigated the performance of an improved dual reflector foldable paraboloid solar cooker, along with its energy and exergy analysis. Their results indicated an average cooking power of $485 \mathrm{~W}$, peak exergy gain of $60.53 \mathrm{~W}$, quality factor of 0.077 , and a high product of temperature difference gap at half peak power to peak power of $4364.33 \mathrm{~W} \cdot \mathrm{K}$. The maximum exergy output power attained was $70 \mathrm{~W}$, while maximum exergy efficiency was $8-10 \%$.

Y. Zhang et al. had two papers discussing the applications of the front-tracking method (FTM) in the heat-absorbing tube of trough solar collector and pulsating heat pipe by considering the multiphase flow and heat transfer.

$\mathrm{W}$. Bao et al. experimentally investigate the phase distribution of subcooled flow boiling in an inclined circular 
tube, and they concluded that the phase distribution under the inclined condition is different from the phase distribution under the vertical condition; more specifically the profiles skewed highly for $90^{\circ}$ and $45^{\circ}$ direction in the tube cross-section, whereas the profile was also symmetrical at $0^{\circ}$ direction.

J. Zhang et al. prepared and characterized the paraffin and EG composite phase change material (PCM), and the composite PCM have been applied in the $42110 \mathrm{LiFePO} 4$ battery module $(48 \mathrm{~V} / 10 \mathrm{Ah})$. The testing results indicated that the PCM cooling system can control the peak temperature under $42^{\circ} \mathrm{C}$ and balance the maximum temperature difference within $5^{\circ} \mathrm{C}$. Even in extreme high-discharge pulse current process, the peak temperature can be controlled within $50^{\circ} \mathrm{C}$.

H. Wang and Q. Yu studied the analytical solution of the vapor flow in rotating heat pipes based on the hypothesis of potential flow, and a specific rotating heat pipe was examined using three different boundary conditions: linear distribution, uniform but asymmetric distribution, and uniform and symmetric distribution of heat load; therefore, the flow field was calculated, and the Coriolis force is estimated.

C. Luo et al. investigated a novel solar PCM storage wall technology, which is a dual-channel and thermalinsulation-in-the-middle type solar PCM storage wall (MSPCMW) system. Basically, the system has four independent functions, passive solar heating, heat preservation, heat insulation, and passive cooling; therefore, this system can agilely cope with the requirements of climatization of buildings in different seasons, and therefore they experimentally analyzed the thermal feature of the system working in two different modes, summer and winter, respectively.

\section{Acknowledgments}

The authors would like to express their appreciation to all the authors and reviewers.

Ben Xu

Juan Ordonez

Zhonghao Rao

Xinhai $X u$

Hamidreza Shabgard

\section{References}

[1] M. Liu, W. Saman, and F. Bruno, "Review on storage materials and thermal performance enhancement techniques for high temperature phase change thermal storage systems," Renewable and Sustainable Energy Reviews, vol. 16, no. 4, pp. 21182132, 2012.

[2] B. Xu, P. Li, and C. Chan, "Application of phase change materials for thermal energy storage in concentrated solar thermal power plants: a review to recent developments," Applied Energy, vol. 160, pp. 286-307, 2015.

[3] J. Pereira da Cunha and P. Eames, "Thermal energy storage for low and medium temperature applications using phase change materials - a review," Applied Energy, vol. 177, pp. 227-238, 2016. 

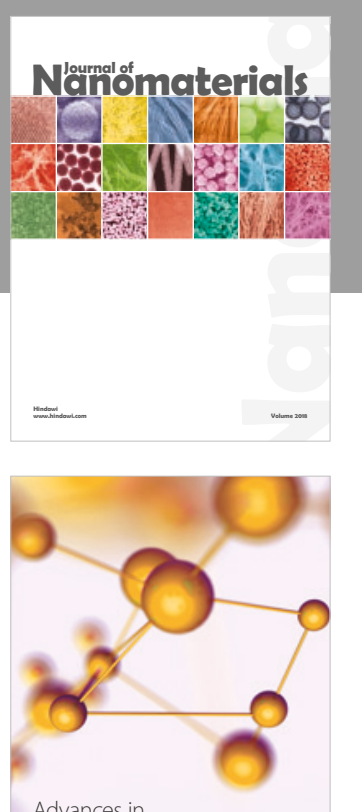

Physical Chemistry
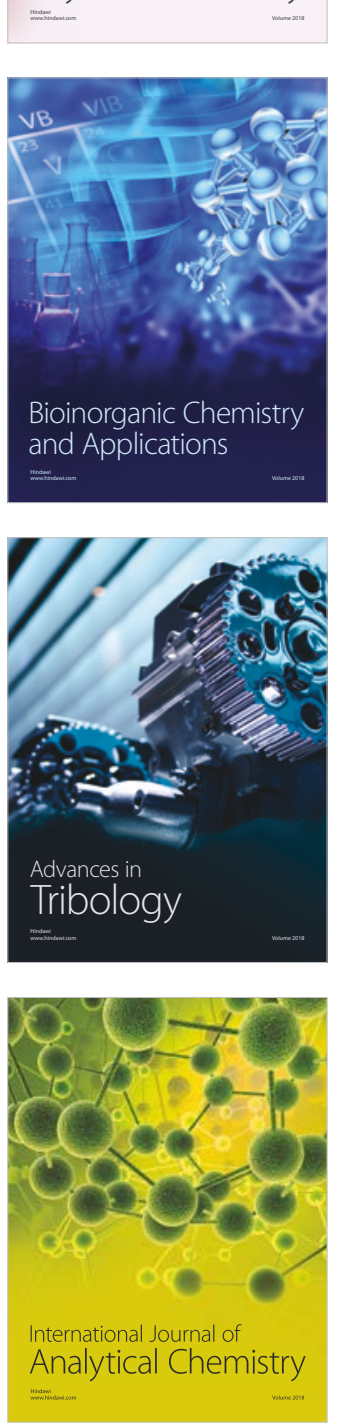

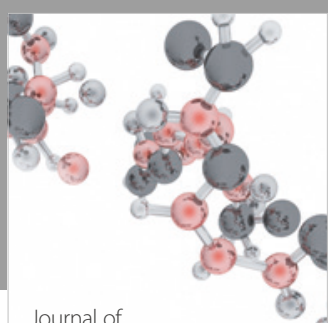

Analytical Methods

in Chemistry

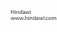

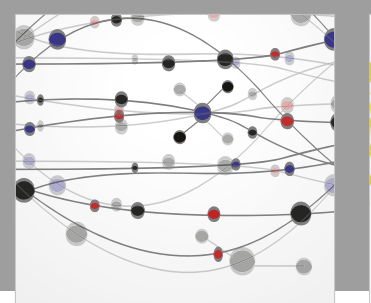

The Scientific World Journal

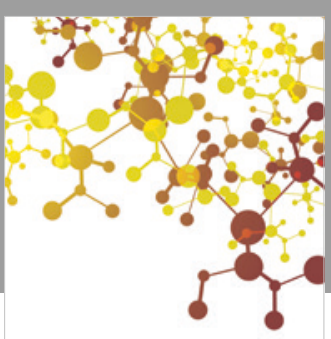

Journal of

Applied Chemistry
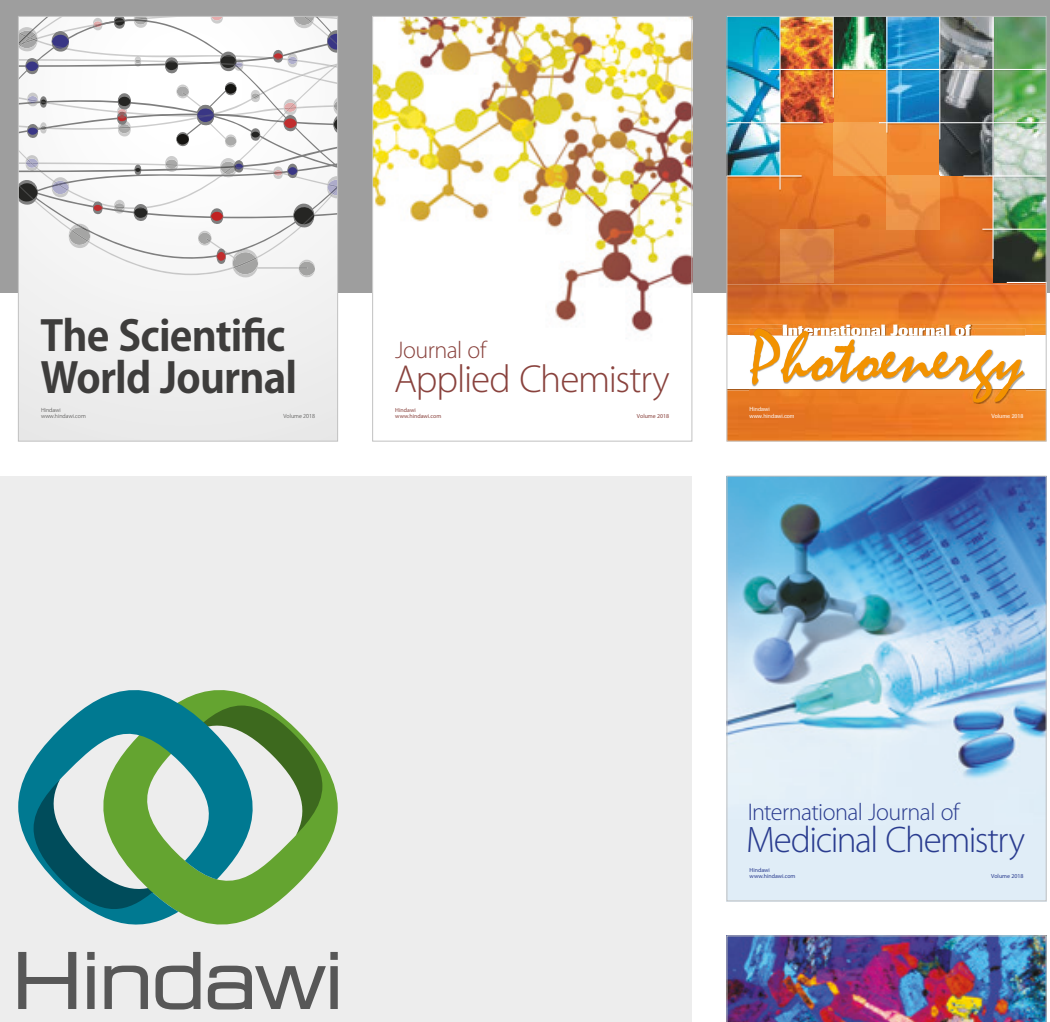

Submit your manuscripts at

www.hindawi.com
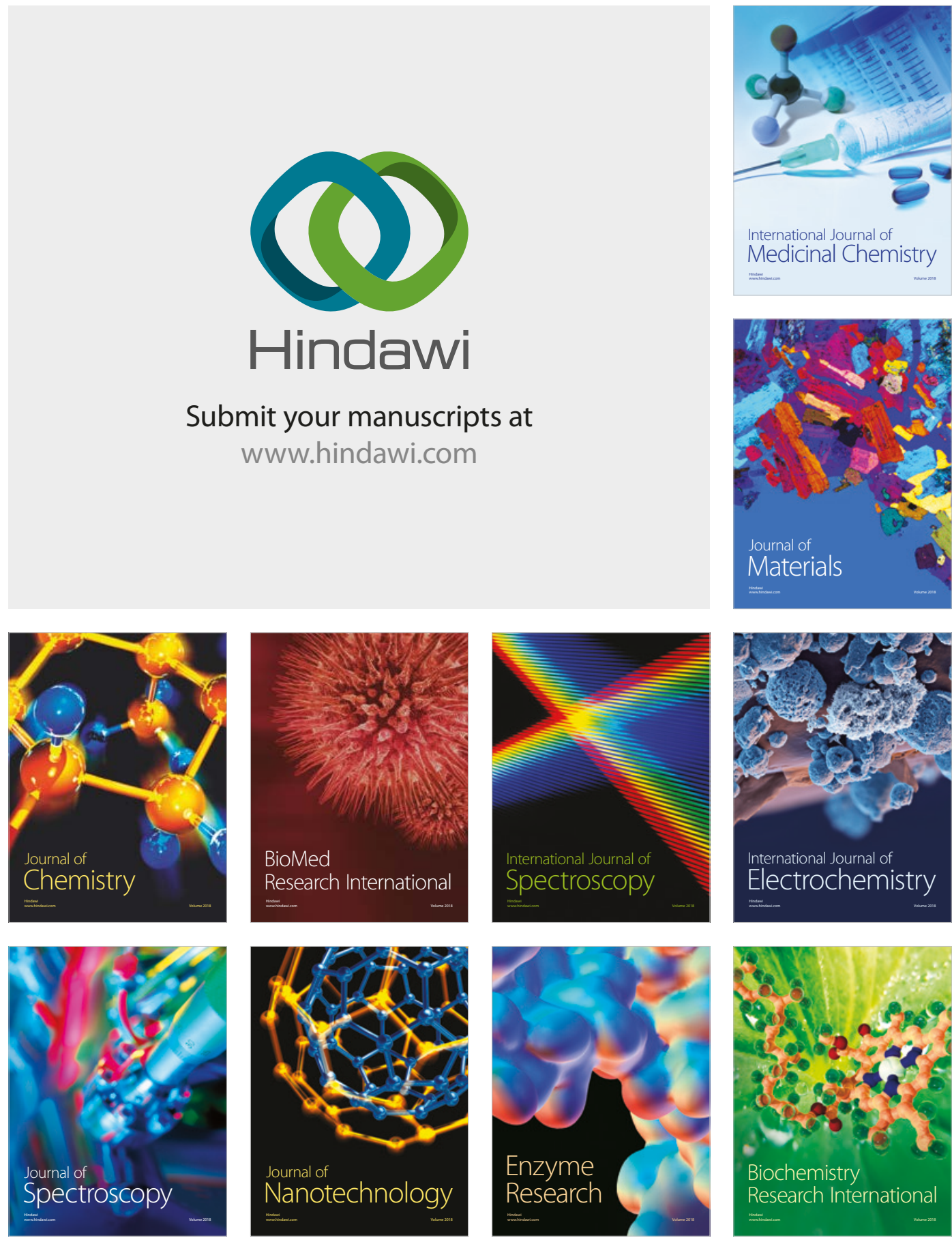
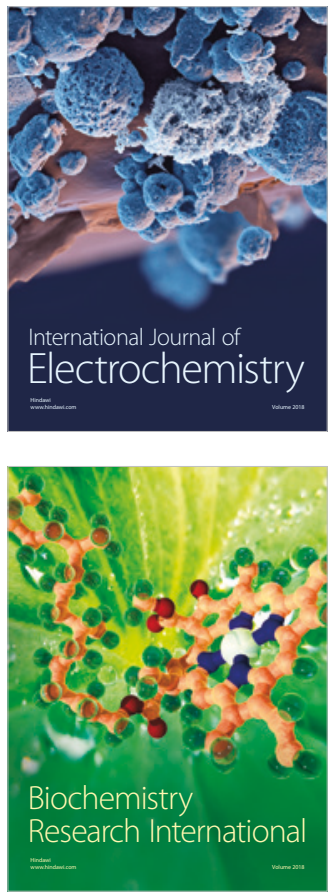